\title{
Scale-free correlations in collective motion with position-based interactions
}

\author{
E. Ferrante ${ }^{1}$, A. E. Turgut ${ }^{1}$, T. Wenseleers ${ }^{1}$, and C. Huepe ${ }^{2}$ \\ ${ }^{1}$ Laboratory for Entomology, Katholieke Universiteit Leuven, 59 Naamsestraat - bus 2466, 3000 Leuven, Belgium \\ ${ }^{2}$ Northwestern Institute on Complex Systems, Northwestern University, Evanston, IL 60208, USA and \\ CHuepe Labs, 954 W. 18th Place, Chicago IL 60608, USA
}

\section{Extended Abstract}

Introduction Collective Motion (CM) is observed in a variety of animal groups such as bird flocks and fish schools. In a recent study, Cavagna et al. (2010) found that the correlation lengths of speed and velocity fluctuations in starling flocks are not set by a specific interaction range, but are instead scale-free, proportional to the group size. So far, this observation has been justified by hypothesizing that flocks evolved to follow critical dynamics near a phase transition, where scale-free correlations are known to emerge. Criticality could provide an evolutionary advantage by allowing the flock to optimally respond to an external perturbation such as a predator attack. However, a criticality-based explanation may only be required in cases where interactions are based exclusively on relative orientations, as often assumed in CM models, following the seminal work by Vicsek et al. (1995). In this paper, we show that an alternative, more parsimonious, mechanism can produce scale-free correlations when considering interactions based on relative positions.

AE simulations We consider an active elasticity (AE) model (Ferrante et al., 2013), where $N$ self-propelled agents are moving in $2 \mathrm{D}$, with neighbors permanently linked by spring-like linear forces. Given the position $\vec{x}_{i}$ and orientation $\theta_{i}$ of each agent $i$, the AE model is defined as:

$$
\begin{aligned}
\dot{\vec{x}}_{i} & =v_{0} \hat{n}_{i}+\alpha\left(\vec{F}_{i} \cdot \hat{n}_{i}\right) \hat{n}_{i}, \\
\dot{\theta}_{i} & =\beta\left(\vec{F}_{i} \cdot \hat{n}_{i}^{\perp}\right)+D_{\theta} \xi_{\theta} .
\end{aligned}
$$

Here, $v_{0}$ is the preferred self-propulsion speed, $\alpha$ and $\beta$ are the speed and angular force coupling coefficients, and $\hat{n}_{i}$ and $\hat{n}_{i}^{\perp}$ are unit vectors pointing parallel and perpendicular to the heading of agent $i$, respectively. The sum of elastic forces over agent $i$ is given by: $\vec{F}_{i}=$ $\sum_{j \in S_{i}}\left(-k / l_{i j}\right)\left(\left\|\vec{r}_{i j}\right\|-l_{i j}\right)\left(\vec{r}_{i j} /\left\|\vec{r}_{i j}\right\|\right)$, where $S_{i}$ is the set of all $j$ neighbors linked to agent $i$, the spring constants $k / l_{i j}$ and natural lengths $l_{i j}$ characterize the interactions between them, and $\vec{r}_{i j}=\vec{x}_{j}-\vec{x}_{i}$ are their relative positions. Noise is introduced by adding $D_{\theta} \xi_{\theta}$ in Eq. (2), where $D_{\theta}$ is the noise strength and $\xi_{\theta}$ a random variable with standard, zero-centered normal distribution of variance 1 .
Simulations were carried out by integrating Eqs. (1) and (2) numerically. They were set up as a minimal AE-based version of the starling experiments by Cavagna et al. (2010). Parameters $l_{i j}=1, v_{0}=10, k=100, \alpha=2$, and $\beta=3$ were chosen to roughly mimic the experimental dynamics. The noise level was set to be high $\left(D_{\theta}=0.628\right)$ but far from any critical point (here at $D_{\theta}^{*} \approx 1.1$, where the system transitions to a disordered, non-flocking state). We focus on the correlations that develop as the group turns due to strong local perturbations. This is continuously occurring in natural flocks as individuals at the edge of the group change their heading directions based on external stimuli. We mimic such situation by initializing all agents with $\theta=0$, except for one (the informed agent), which is forced to head towards $\theta=$ $\pi / 3$ during the whole run, while its speed is determined by Eq. (1). This corresponds roughly the natural flock turning dynamics described in (Attanasi et al., 2013).

Figure 1 displays snapshots of the AE simulations. At $t=0.5$ (a), the top-right corner of the hexagon is turning towards $\theta=\pi / 3$, as imposed by the informed agent, while the rest continues heading to the right, still unperturbed. In the subsequent $t=1.0$ (b), 1.5 (d), and 2.0 (e) snapshots, the whole group starts to acquire the $\pi / 3$ orientation, as the local perturbation spreads out through the system. Despite the turning and imposed noise, the degree of agent alignment remains high, with polarization order parameter $\psi=\left\|\sum_{i=1}^{N} \vec{v}_{i}\right\| /\left(N v_{0}\right)$ ranging between 0.90 and $0.96(\psi=1$ indicates full alignment), which is consistent with the starling experiments. All panels display the informed agent detached from the hexagon, above and to the left of its top-right corner, since its virtual springs sustain large forces as they induce group turning, and are therefore very stretched.

Correlation function analysis We focus on the speed fluctuations $s_{j}=\left\|\vec{v}_{j}\right\|-\frac{1}{N} \sum_{i=1}^{N}\left\|\vec{v}_{i}\right\|$ and velocity fluctuations $\vec{u}_{j}=\vec{v}_{j}-\frac{1}{N} \sum_{i=1}^{N} \vec{v}_{i}$. As in (Cavagna et al., 2010), we compute the correlation function for the latter using

$$
C_{\vec{u}}(r)=K \frac{\sum_{i, j=1}^{N} \vec{u}_{i} \cdot \vec{u}_{j} \delta\left(r-r_{i j}\right)}{\sum_{i, j=1}^{N} \delta\left(r-r_{i j}\right)},
$$




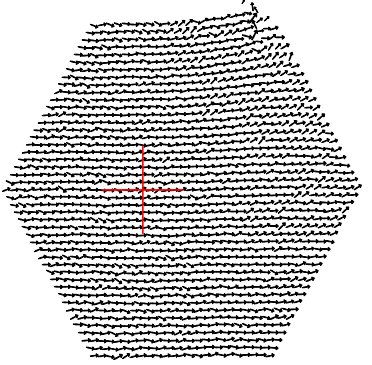

(a)

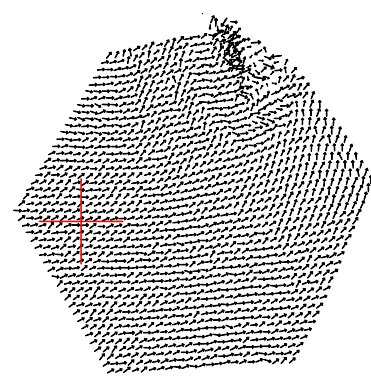

(d)

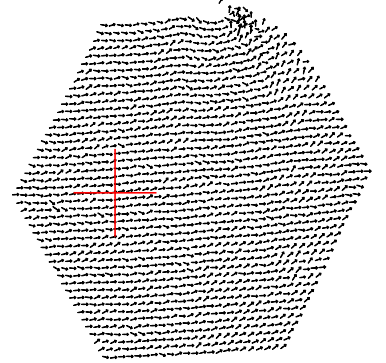

(b)

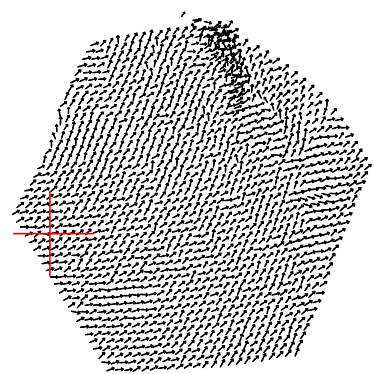

(e)

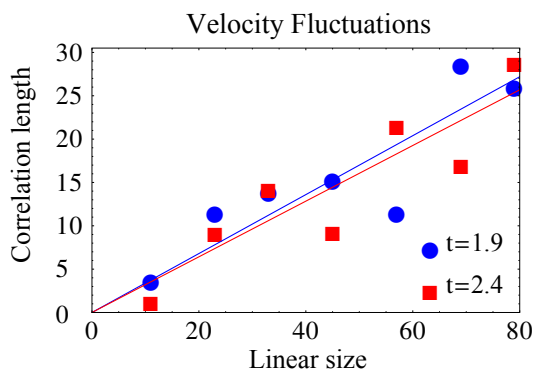

(c)

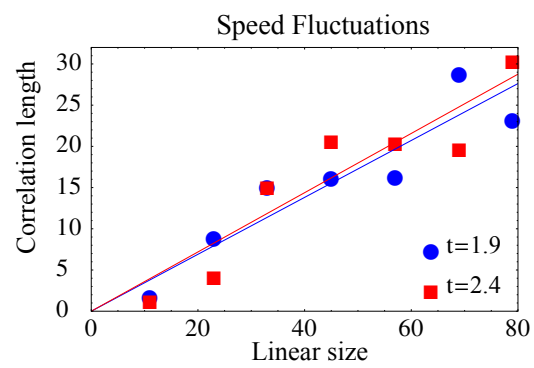

(f)

Figure 1: Simulation snapshots at $t=0.5$ (a), 1.0 (b), 1.5 (d), and 2.0 (e). Red crosses indicate where the hexagon's center was at $t=0$. Correlation lengths of the velocity (c) and speed fluctuations (f) as a function of group sizes at two different times.

and the correlation function $C_{s}(r)$ for the speed fluctuations using an equivalent expression, but where $\vec{u}_{i}$ is replaced by $s_{i}$ and the dot product by multiplication. Here, $r_{i j}$ is the distance between agents $i$ and $j, \delta(\cdot)$ is a smoothed Dirac delta function, and $K$ is a normalizing constant defined so that $C_{\vec{u}}(0)=1$. Both functions must cross zero since $s_{i}$ and $\vec{u}_{i}$ have zero mean. We thus define as correlation lengths the first zero-crossing points of $C_{\vec{u}}(r)$ and $C_{s}(r)$.

We performed the simulations and analysis for hexagonal groups containing $N=91,397,817,1519,2437,3571$, and 4681 agents, a range slightly larger than in the starling experiments. Correlation functions were computed at $t=1.9$ and 2.4 (two somewhat arbitrary instants, chosen slightly after the perturbation crosses our largest hexagon) in order to examine the typical correlation lengths and their degree of variation. Panels (c) and (f) display the correlation lengths of the velocity and speed fluctuations, respectively, as a function of the linear size of the group, defined here as the distance between opposing vertices of the undeformed hexagon. While we observed significant variations throughout the dynamics, both quantities are shown to be large and proportional to the group size, as in Cavagna et al. (2010).

Discussion and conclusion The results above show that the scale-free correlations observed in starling experiments are not necessarily due to a critical regime, but can be a natural consequence of position-based interactions among in- dividuals. Our work also provides a bio-inspired algorithm that can produce coherent, group-level collective motion for robot swarms or other artificial flocks. By implementing the AE model, highly correlated motion dynamics that always span a significant and approximately constant fraction of the group, regardless of its size, can be achieved.

Acknowledgements This work was partially supported by the Vlaanderen Research Foundation Flanders (H2Swarm project), the US National Science Foundation (Grant No. PHY-0848755) and TUBITAK (Grant No. 2219).

\section{References}

Attanasi, A., Cavagna, A., Del Castello, L., Giardina, I., Grigera, T. S., Jelic, A., Melillo, S., Parisi, L., Pohl, O., Shen, E., and Viale, M. (2013). Superfluid transport of information in turning flocks of starlings. Cond-mat.stat-mech, arXiv:1303.7097.

Cavagna, A., Cimarelli, A., Giardina, I., Parisi, G., Santagati, R., Stefanini, F., and Viale, M. (2010). Scale-free correlations in starling flocks. Proceedings of the National Academy of Sciences, 107:11865-11870.

Ferrante, E., Turgut, A. E., Dorigo, M., and Huepe, C. (2013). Collective motion dynamics of active solids and active crystals. New Journal of Physics, 15(9):095011.

Vicsek, T., Czirok, A., Ben-Jacob, E., Cohen, I., and Shochet, O. (1995). Novel type of phase transition in a system of selfdriven particles. Physical Review Letters, 75(6):1226. 